\title{
Eco-evolutionary feedbacks in the human gut microbiome
}

\author{
Benjamin H. Good ${ }^{1, \dagger}$, Layton B. Rosenfeld ${ }^{1}$ \\ ${ }^{1}$ Department of Applied Physics, Stanford University, Stanford, CA 94305, USA \\ †Correspondence should be addressed to: B.H.G. (bhgood@stanford.edu)
}

Gut microbiota can evolve within their hosts on human-relevant timescales. Yet little is known about how these changes influence (or are influenced by) the composition of their surrounding community. Here, by combining ecological and evolutionary analyses of a large cohort of human gut metagenomes, we show that the short-term evolution of the microbiota is linked with shifts in its ecological structure. These correlations are not simply explained by expansions of the evolving species, and often involve additional fluctuations in distantly related taxa. We show that similar feedbacks naturally emerge in simple resource competition models, even in the absence of cross-feeding or predation. These results suggest that the structure and function of host microbiota may be shaped by their local evolutionary history, with potential implications for personalized medicine and disease.

\section{Main Text}

The human gut harbors a diverse microbial community comprising hundreds of ecologically interacting strains $(1,2)$. Recent work has shown that the residents of this community can also evolve over time, through a mixture of within-host evolution (3-9) and the invasion of external strains $(4,6,10)$. In principle, these rapid genetic changes could alter ecological interactions between species, driving shifts in community composition, and spurring further co-evolutionary responses in other resident strains. Yet despite intensive theoretical speculation (11-16), little is currently known about how the short-term evolution of the microbiota influences (or is influenced by) the composition of its local community.

Previous work has shown that ongoing evolution can alter the ecological composition of small in vitro communities (17-20). However, it remains unclear how this behavior generalizes to complex ecosystems like the gut microbiota. Experiments have shown that the disruption of particular metabolic pathways can alter crossfeeding interactions between certain species of gut bateria $(21,22)$, while other variants can enable the colonization of open metabolic niches (23). However, it is not known how often such mutations are selected in in situ, or whether the niche partitioning (24) or functional redundancy $(1,25)$ of native gut communities tends to shield them from such evolutionary perturbations.

To distinguish these scenarios, we reanalyzed a large collection of fecal metagenomes from the Human Microbiome Project $(1,2)$, which followed $>100$ healthy human subjects at 2-3 timepoints over a period of $\sim 6$ months (Table S1). We hypothesized that this large cohort would provide an opportunity to measure eco-evolutionary feedbacks at a statistical level, by asking whether within-host evolution tends to be accompanied by larger shifts in taxonomic composition during the same time interval. Since gut microbiota also experience daily fluctuations in the absence of evolution $(6,26-28)$, this approach requires us to search for global patterns that exceed this baseline variation.

To carry out this analysis, we used a reference-based approach (4) to identify single nucleotide variants (SNVs) that underwent large shifts in frequency within a host between pairs of sequenced 
timepoints (Methods). These large frequency changes indicate a partial or complete "sweep" within the species in question, in which the focal SNV is likely hitchhiking as a linked passenger mutation (9). We further classified the sweeps within each species as evolutionary modification or strain replacement events (Fig. S1) based on the total number of correlated SNV changes along that species' genome (Methods); we previously showed that the strain replacement events are accompanied by large differences in gene content ( $\sim 100-1000$ 's of genes), comparable to other circulating strains in the global human population (4). Using these methods, we compiled a dataset of 16 replacement events and 78 modifications from 799 pairwise genetic comparisons across 45 different species and 134 unique hosts (Table S2). These data provide a unique opportunity to quantify the links between short-term evolution and community structure in the native human gut microbiota in its complex natural environment.

We first asked how the rates of within-species evolution varied with the composition of the local community. We found that the strain replacement and evolutionary modification events were both non-uniformly distributed across taxa. While the sample sizes were too small to resolve the rates of individual species, an omnibus test still revealed a global enrichment of variability across species $\left(P \approx 0.01\right.$, Methods), with even stronger signals at the family or phylum levels $\left(P<10^{-4} ;\right.$ Fig. $\left.1 \mathrm{~A}, \mathrm{~B}\right)$. Much of this signal was driven by differences between the Bacteroidetes and Firmicutes phyla, with the latter experiencing replacement and modification events at $\sim 3$-fold higher rates (Fig. 1A,B).

We also investigated how the strain replacement and evolutionary modification events depended on the global properties of their surrounding community. For example, classical theories suggest that the rates of evolution may strongly depend on local species diversity $(29,30)$. We found that evolutionary modification events were weakly correlated with the Shannon diversity at the initial timepoint, after controlling for differences between phyla ( $P \approx 0.04$; Methods; Fig. $1 \mathrm{C}$, Fig. S2). However, this residual dependence was much smaller than the differences between phyla, and was primarily driven by the top decile of the Bacteroidetes distribution (rather than a systematic trend across all samples). Consistent with this observation, we observed no strong correlations between replacement and modification events in different species in the same host (Fig. 1D), as might be expected if the genetic changes were primarily determined by a global property of the community like diversity. These results suggest that to a first approximation, the genetic changes within our cohort can be described by a simple null model, in which replacement and modification events occur approximately independently in different resident populations, with species-specific rates.

Armed with this information, we next asked how the genetic changes within species were correlated with shifts their in ecological structure over time. Within our cohort, one can find individual hosts with replacement or modification events that were also accompanied by large changes in species composition between the same two timepoints (Fig. 2A,B). One can also find hosts with no detected genetic changes that experienced smaller ecological shifts (Fig. 2C). To quantify this pattern more systematically, we partitioned our 194 community comparisons into three categories depending on whether the community had at least one replacement event, at least one modification event, or no genetic changes within species (Fig. 2D). For each of these communities, we also calculated the Jensen-Shannon (JS) distance between the species abundance distributions at the initial and final timepoints (Methods); this provides a simple metric for quantifying the overall change in species-level composition over time (31).

While species abundances can fluctuate due to a variety of intrinsic and extrinsic factors $(6,26-28)$, we observed a small but systematic trend toward larger JS distances in hosts that experienced an 

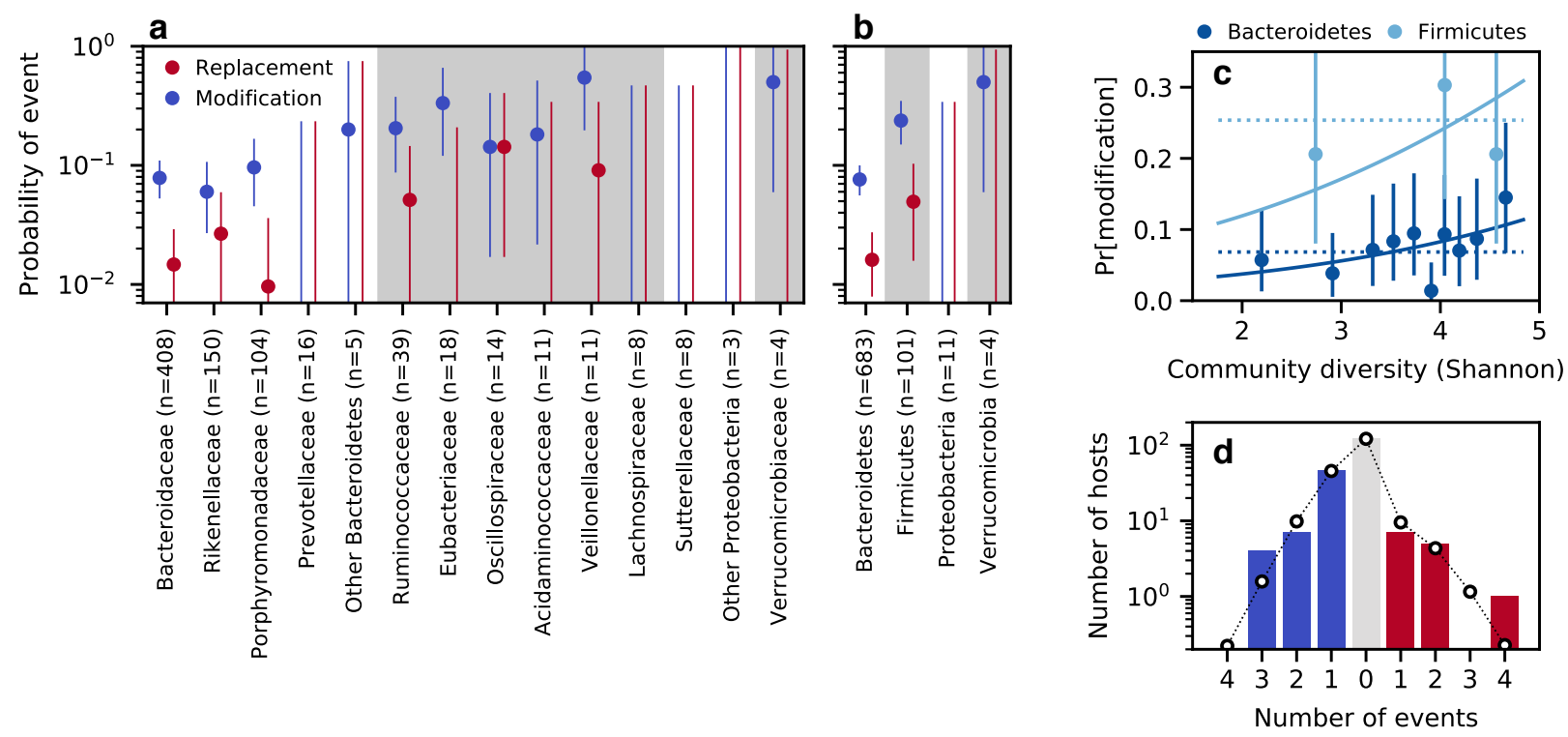

Figure 1: Replacement and modification rates vary across species, but only weakly depend on the diversity of the surrounding community. (a,b) Fraction of resident populations in which we detected strain replacement or evolutionary modification events (Methods), coarse-grained at the family (a) or phylum level (b). Lines denote $95 \%$ confidence intervals based on the observed number of counts (Methods). (c) The probability of a modification event as a function of the species diversity of the surrounding community. Points show the fraction of modification events in Bacteroidetes or Firmicutes populations stratified by quantiles of the Shannon diversity; 95\% confidence intervals were computed as above. Solid lines illustrate the best-fit logistic regression model using the phylum and community diversity as predictor variables. For comparison, the dashed lines show the average of each phylum computed for the bottom $90 \%$ of community diversity values (the diversity term is no longer significant in this case, $P \approx 0.2$ ). (d) The distribution of the number of genetic changes per community. Bars denote the fraction of community comparisons with a given number of genetic events, partitioned into communities with at least one replacement event (right) and communities that only experienced evolutionary modification events (left). Lines denote the null distribution obtained by randomly permuting replacement and modification events across resident populations of the same species (Methods). 

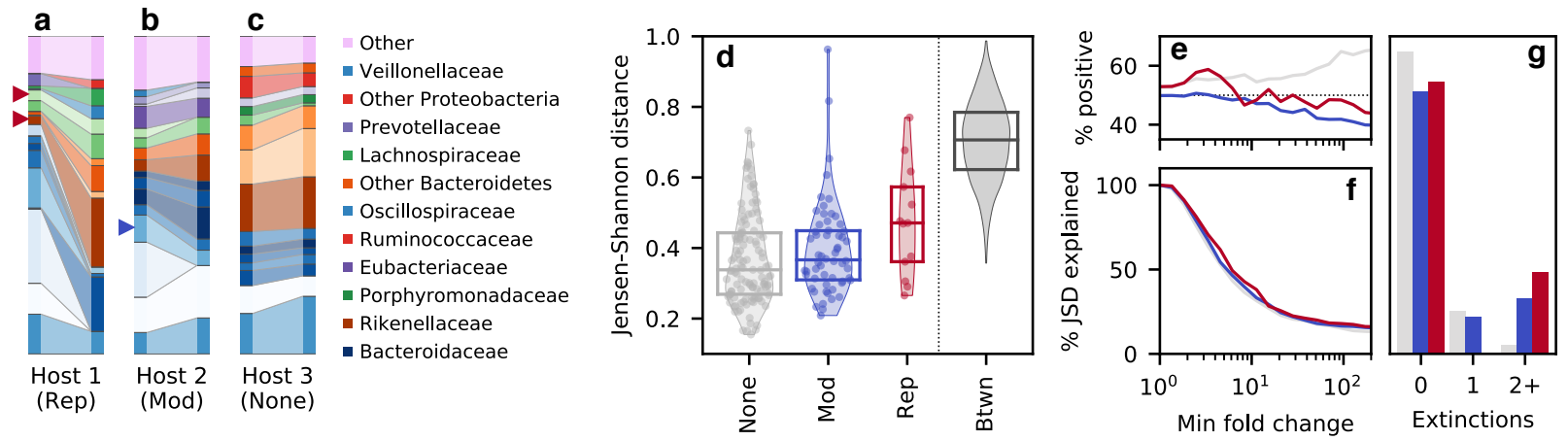

Figure 2: Genetic changes within species are statistically associated with shifts in taxonomic composition over time. (a-c) Species relative abundances over time in three example hosts experiencing strain replacement events (a), evolutionary modification events (b), and no genetic changes (c). Species are shown if they had a relative abundance $>2 \%$ in one of the hosts in at least one timepoint; all other species are grouped together in the 'Other' category. Species experiencing replacement and modification events are indicated by the red and blue triangles, respectively. (d) Distribution of JensenShannon distances over time (Methods) for communities that experienced at least one replacement (Rep) or modification event (Mod), or no genetic changes (None). Symbols denote individual data points, while box plots show the median and inter-quartile range; the JS distances between $\sim 1000$ random pairs of hosts are shown at right for comparison. $(e, f)$ For each of the communities in panel $d$, the fraction of the Jensen-Shannon divergence explained by fold changes greater than a given amount (f, Methods), as well as the fraction of this amount explained by positive vs negative changes (e). ( $g$ ) The number of abundant species that went extinct in each of the communities in panel $d$ (Methods). 
evolutionary modification event, and even larger distances in hosts that experienced a strain replacement event (Fig. 2D). To assess the significance of these trends, we utilized an empirical null model informed by Fig. 1: we generated $n=10^{4}$ bootstrapped datasets by randomly permuting the observed genetic events within the resident populations of each species. By construction, these bootstrapped datasets preserve both the overall number of genetic changes of each type, their distribution across species, and the species abundance trajectories within each host. This provides a principled way to test for associations between ecological and genetic changes, while preserving the complex correlations between the abundances of different species in large microbial communities.

We found that both replacement and modification events have significantly larger JS distances than expected under this empirical null model $(P \approx 0.001$ and $P \approx 0.003$ respectively; Methods), suggesting that the ecological and genetic changes within these communities are indeed correlated each other. Notably, the evolutionary modification signal is primarily driven by a depletion of the smallest temporal fluctuations (Fig. S4), rather than the handful of hosts with the largest ecological shifts. By contrast, the strain replacement signal is driven by a global increase in JS distances (Fig. S4), which could be consistent with both an increased power to detect these events, as well as the larger number of SNV and gene-content differences that they contribute. Similar differences are observed when community composition is measured at the genus, family, or phylum levels (Fig. S3). We also observed a small but significant enrichment of "extinction" events in abundant species (modifications $P \approx 0.003$, replacements $P \approx 0.01$; Methods) (Fig. 2G), which suggests that these eco-evolutionary correlations are not specific to the Jensen-Shannon metric.

The ecological distances in Fig. 2D can be further decomposed into the contributions from different species (Methods). Interestingly, this decomposition shows that the larger distances in Fig. 2 are not solely driven by expansions of the focal species (i.e., those with genetic changes), as expected under some of the simplest models of niche expansion. While we observed a small bias toward expansions in species that experienced a modification event $(P \approx 0.05$, Methods), the focal species still declined in frequency in more than $40 \%$ of cases (Fig. 3A,B). Strain replacements displayed a similar trend: while the focal species experienced significantly larger fold-changes changes over time $(P \approx 0.03$, Methods), the majority of these changes involved declines in relative abundance rather than expansions (Fig. 3A,B). Furthermore, we observed no strong correlation between the community-level distances in Fig. 2D and the fold changes in the focal species (Fig. 3D). This suggests that the eco-evolutionary correlations in Fig. 2D do not depend on the direction of the focal species's trajectory. Rather, the observed ecological shifts are more global in nature, in that they are comprised of correlated shifts in the abundances of other species in the community, even when focal species itself declines.

Fig. 2B illustrates a prototypical example of this behavior: we detected an evolutionary modification event in one species (Bacteroides stercoris), which declined slightly in relative abundance, while two other species went extinct in the same time interval - one from the same genus (Bacteroides massiliensis) and another from a different bacterial family (Barnesiella intestinihominis). To quantify the relationships between these species more systematically, we calculated the fraction of JSD that contributed by species in the same family as one of the focal species (Fig. 3F). We found that the family-level contributions were somewhat larger than the contributions of the focal species themselves (Fig. 3G), consistent with the larger number of species involved, but they were not significantly different than expected by chance ( $P \approx 0.07$; Methods). This shows that the statistical signal in Fig. 2 is not solely driven by fluctuations in closely-related species, but rather by correlated fluctuations in distantly-related taxa. 
a

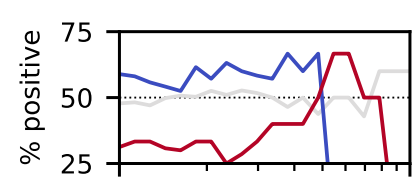

b

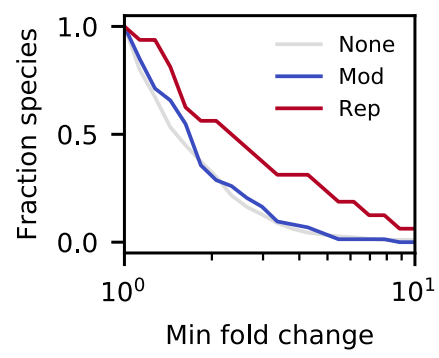

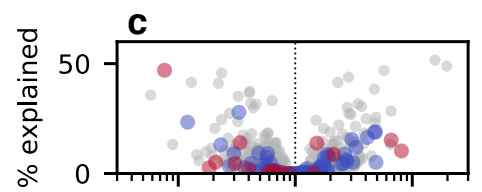

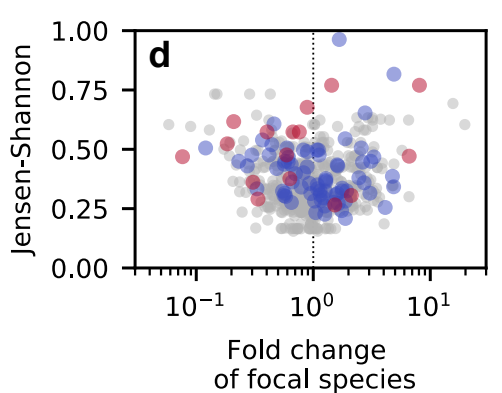

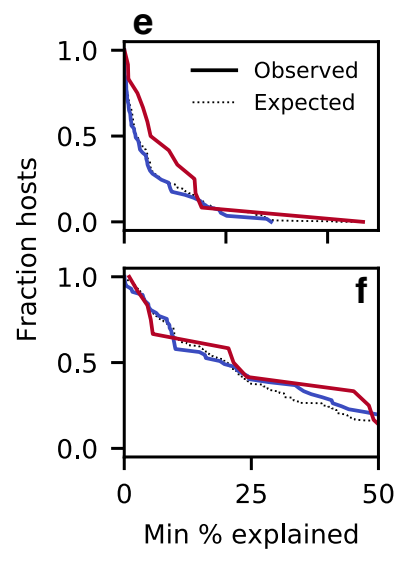

Figure 3: Ecological changes are not solely explained by frequency increases in focal species. $(a, b)$ Fraction of focal species with fold changes in relative abundance greater than a given amount (b), and the fraction of this amount contributed by positive vs negative changes (a). (c,d) The JensenShannon distances in Fig. 2D as a function of the fold change in relative abundance of the focal species (d), and the fraction of the Jensen-Shannon divergence explained by each of these fold changes (c, Methods). (e) The fraction of community comparisons in which the Jensen-Shannon divergence explained by the focal species exceeds a given percentage. Solid lines denote the observed data for the replacement and modification hosts in Fig. 2D, while the dashed lines denote the expectations of a null model where the focal species are chosen at random (Methods). (f) An analogous version of panel e using the fraction of Jensen-Shannon divergence explained by all species in the same family as one of the focal species. 
Our results establish a statistical link between the short-term evolution of the human gut microbiota and changes in its ecological structure. Such correlations could arise through several causal scenarios: genetic changes could alter ecological interactions between species, driving shifts in community composition (evolution-driven feedbacks); alternatively, environmental perturbations could lead to taxonomic shifts while also creating new opportunities for within-host evolution (ecology-driven feedbacks). It is difficult to distinguish these scenarios from observational data alone, though the independent distribution of genetic events (Fig. 1D) and the slight bias toward the expansion of focal species (Fig. 2D) may provide some evidence in favor of the evolution-driven scenario.

To gain more intuition for the range of possible behaviors, we also studied the correlations between ecological and genetic changes in a simple mathematical model, where the contributions of these two feedback mechanisms could be precisely controlled. We considered a simple class of resource competition models (32), in which $\mathcal{S}$ coexisting species compete for $\mathcal{R}$ substitutable resources that are continuously supplied by the environment $(15,33)$ (Text S1). For a saturated community $(\mathcal{S}=\mathcal{R})$ at ecological equilibrium, the selection pressures on new mutations are independent of the external environment and the abundances of the resident species (15). This constitutes a simple example in which ecology-driven feedbacks are effectively damped by the collective response of the community. In this case, one can derive a further result connecting the invasion fitness of a small-effect mutation with the ecological perturbations that it causes after it invades:

$$
S_{\mathrm{inv}} \propto\left[\Delta X_{\mu^{*}} \cdot f_{\mu^{*}}+\sum_{\mu=1}^{\mathcal{S}} \Delta f_{\mu} \cdot X_{\mu}\right] .
$$

Here $f_{\mu}$ denotes the relative abundance of species $\mu, X_{\mu}$ is the logarithm of its total resource uptake rate, and $\mu^{*}$ denotes the focal species in which the mutation occurred (Text S1). In this case, we see that the invasion fitness of a mutation depends on its future ecological impact only through the community-level quantity $\sum_{\mu} \Delta f_{\mu} X_{\mu}$, which is independent of $\mu^{*}$, and is also independent of the metabolic phenotypes of the resident strains. This shows that it is possible for natural selection to favor mutations that lower the abundance of the focal species $\left(\Delta f_{\mu^{*}}<0\right)$, providing a potential mechanism for the empirical behavior observed in Fig. 3A. It also shows that mutations can produce a mixture of positive and negative abundance changes across a large range of species, regardless of their phenotypic similarity.

Together, these results show that simple evolution-driven feedbacks can recapitulate some of the qualitative features of Figs. 2 and 3, even in the absence of additional factors like crossfeeding $(34,35)$, spatial structure (36), or phage predation (37). Further work will be required to determine which of these ecological or evolutionary feedback mechanisms are most relevant for the human gut, and to map out the empirical landscape of $\left(S_{\mathrm{inv}},\left\{\Delta f_{\mu}\right\}\right)$ values that can be produced by mutating or swapping resident strains. Our results show that the statistical signature of these feedbacks can sometimes be observed even in complex in situ environments, generating targeted hypotheses that could be tested in future experiments, e.g. using large synthetic communities (38). These results could have important implications for the development of personalized therapies that aim to tune the composition of the gut microbiota. Generalizations of our approach could be useful for quantifying the links between short-term evolution and community structure in other microbial ecosystems that are characterized by large numbers of coexisting species (39-42). 


\section{References}

[1] C. Huttenhower, et al., Nature 486, 207 (2012).

[2] J. Lloyd-Price, et al., Nature 550, 61 (2017).

[3] M. Ghalayini, et al., Appl. Environ. Microbiol. 84, e02377 (2018).

[4] N. R. Garud, B. H. Good, O. Hallatschek, K. S. Pollard, PLoS biology 17, e3000102 (2019).

[5] S. Zhao, et al., Cell host \& microbe 25, 656 (2019).

[6] M. Poyet, et al., Nature medicine 25, 1442 (2019).

[7] E. Yaffe, D. A. Relman, Nature Microbiology 5, 343 (2020).

[8] S. Zlitni, et al., Genome Medicine 12, 50 (2020).

[9] M. Roodgar, et al., Genome research 31, 1433 (2021).

[10] B. A. Siranosian, et al., bioRxiv (2021).

[11] K. R. Foster, J. Schluter, K. Z. Coyte, S. Rakoff-Nahoum, Nature 548, 43 (2017).

[12] A. Ferreiro, N. Crook, A. J. Gasparrini, G. Dantas, Cell 172, 1216 (2018).

[13] G. Ackland, I. Gallagher, Phys Rev Lett 93, 158701 (2004).

[14] N. Shoresh, M. Hegreness, R. Kishony, Proc Natl Acad Sci USA 105, 12365 (2008).

[15] B. H. Good, S. Martis, O. Hallatschek, Proceedings of the National Academy of Sciences 115, E10407 (2018).

[16] L. Fant, I. Macocco, J. Grilli, bioRxiv (2021).

[17] B. H. Good, M. J. McDonald, J. E. Barrick, R. E. Lenski, M. M. Desai, Nature 551, 45 (2017).

[18] N. Meroz, N. Tovi, Y. Sorokin, J. Friedman, Nature communications 12, 1 (2021).

[19] J. N. Barber, et al., The ISME Journal 15, 746 (2021).

[20] S. Venkataram, H.-Y. Kuo, E. F. Hom, S. Kryazhimskiy, bioRxiv (2021).

[21] S. Rakoff-Nahoum, K. R. Foster, L. E. Comstock, Nature 533, 255 (2016).

[22] J. Feng, et al., Cell host \& microbe (2022).

[23] E. S. Shepherd, W. C. DeLoache, K. M. Pruss, W. R. Whitaker, J. L. Sonnenburg, Nature 557, 434 (2018).

[24] S. Brochet, et al., bioRxiv (2021).

[25] L. Tian, et al., Nature communications 11, 1 (2020).

[26] L. A. David, et al., Genome biology 15, R89 (2014).

[27] A. J. Johnson, et al., Cell host \& microbe 25, 789 (2019). 
[28] B. W. Ji, R. U. Sheth, P. D. Dixit, K. Tchourine, D. Vitkup, Nature microbiology 5, 768 (2020).

[29] R. H. Whittaker, Taxon 21, 213 (1972).

[30] D. L. Rabosky, A. H. Hurlbert, The American Naturalist 185, 572 (2015).

[31] S. P. Preheim, et al., Methods in enzymology 531, 353 (2013).

[32] D. Tilman, Resource competition and community structure (Princeton University Press, 1982).

[33] R. Mac Arthur, Proceedings of the National Academy of Sciences 64, 1369 (1969).

[34] T. Wang, A. Goyal, V. Dubinkina, S. Maslov, PLoS Computational Biology 15 (2019).

[35] R. Marsland III, et al., PLoS computational biology 15, e1006793 (2019).

[36] O. M. Ghosh, B. H. Good, bioRxiv (2021).

[37] M. T. Pearce, A. Agarwala, D. S. Fisher, Proceedings of the National Academy of Sciences 117, 14572 (2020).

[38] A. G. Cheng, et al., bioRxiv (2021).

[39] J. E. Goldford, et al., Science 361, 469 (2018).

[40] A. Goyal, L. S. Bittleston, G. E. Leventhal, L. Lu, O. X. Cordero, BioRxiv (2021).

[41] T. Scheuerl, et al., Nature communications 11, 1 (2020).

[42] Y. Bai, et al., Nature 528, 364 (2015).

[43] S. Nayfach, B. Rodriguez-Mueller, N. Garud, K. S. Pollard, Genome Res 26, 1612 (2016).

[44] S. Seabold, J. Perktold, 9th Python in Science Conference (2010).

[45] M. Tikhonov, R. Monasson, Physical review letters 118, 048103 (2017).

[46] A. Posfai, T. Taillefumier, N. S. Wingreen, Physical review letters 118, 028103 (2017).

[47] P. Chesson, Theoretical Population Biology 37, 26 (1990).

[48] A. Eyre-Walker, P. D. Keightley, Nature Reviews Genetics 8, 610 (2007).

\section{Acknowledgments}

We thank Z. Liu for identifying a host mislabeling event in the HMP data, and S. Walton for feedback on the manuscript. This work was supported in part by the Alfred P. Sloan Foundation grant FG-2021-15708 (to B.H.G.) and a Bio-X Undergraduate Research Fellowship (to L.B.R.).

Author contributions: Conceptualization: B.H.G.; theory and methods development: B.H.G. and L.B.R.; analysis: B.H.G. and L.B.R.; writing: B.H.G.

Competing interests: None declared. 
Data and materials availability: Postprocessed data described in the paper are presented in the supplementary materials. Raw sequencing data from the Human Microbiome Project are publicly available at the NCBI Sequence Read Archive using the accessions provided in the supplementary materials. All analysis code is available on Github

(https://github.com/bgoodlab/microbiome_ecoevo_correlations).

\section{Supplementary Materials}

Materials and Methods

Table S1 - S2

Fig S1 - S6

Text $\mathrm{S1}$ 


\section{Materials and Methods}

\section{Metagenomic pipeline.}

We utilized a collection of shotgun metagenomic data from the Human Microbiome Project $(1,2)$ that we analyzed in a previous study (4). This cohort contained a total of 553 fecal samples from 249 healthy human subjects, many of whom were sequenced at 2-3 timepoints roughly 6 months apart (Table S1). We chose to focus on this cohort for several reasons: (i) the samples were sequenced with relatively high coverage, which enhances our ability to detect evolutionary changes within species; (ii) the subjects were specifically selected to avoid unusual external perturbations (e.g. antibiotics, chronic diseases, or large dietary shifts) that could have an outsized influence on microbiome composition; and (iii) we previously showed (4) that the sampling interval in this cohort results in a mixture of hosts with genetic changes in some of their resident species, and other hosts with no genetic changes; this mixture of outcomes is critical for our present analysis.

We processed these data using the pipeline described in Ref. (4). Briefly, we used the MIDAS software package (43) to align the raw sequencing reads from each sample to a large panel of reference genomes representing different species. The relative abundance of each species was estimated in each sample based on the coverage of single copy marker genes. These relative abundances were used to assemble a personalized panel of reference genomes for each host, which were used to identify single nucleotide variants within each species. These initial SNVs were subsequently filtered based on their absolute and relative coverage, as well as their location along the genome, using the same procedures and parameters described in Ref. (4).

\section{Detecting replacement and modification events within species}

We used these data to detect replacement and modification events between pairs of timepoints using the methods described in Ref. (4). For each pair of time points, we identified the subset of "quasi-phaseable" species where the lineage structure was sufficiently simple that the dominant lineage could be identified with a high degree of confidence (4). Within each of these quasi-phaseable populations, we calculated the total number of SNVs that transitioned from $<20 \%$ frequency in one timepoint to $>80 \%$ frequency in another (or vice versa), along with the corresponding number of changes expected from sequencing noise alone (4). As in Ref. (4), we recorded zero SNV differences if the estimated false positive rate was $>10 \%$. The resulting number of SNV differences in each of the populations are listed in Table S2. These counts were used to classify each population as experiencing a replacement event (>100 SNV differences), a modification event (<100 SNV differences), or zero genetic changes; we previously showed that this divergence-based definition is consistent with the patterns of private marker SNV sharing on these time scales (4) (Fig. S1). This analysis yielded a total of 18 replacement events and 98 modification events from a total of 937 pairwise comparisons across 45 different species in 136 unique hosts.

Since some hosts were sampled at more than two timepoints, we de-replicated these data to ensure that all genetic comparisons were performed on non-overlapping time intervals. For each host-species combination, we only included pairwise comparisons from consecutive quasi-phaseable timepoints; all other pairwise comparisons were treated as missing data (similar to non-quasi-phaseable timepoints). This de-replication procedure yielded a final dataset containing 16 replacement events and 78 modification events from a total of 799 pairwise comparisons across 45 different species in 134 unique hosts. These data were used for all of our subsequent analyses. 


\section{Quantifying heterogeneity in replacement and modification rates across species}

We quantified the overall variability in the rates of replacement and modification events across species using a global likelihood ratio test,

$$
\Lambda \equiv \sum_{\mu, e} n_{\mu, e} \log \left(\frac{p_{\mu, e}}{\bar{p}_{e}}\right),
$$

where $n_{\mu, e}$ is the total number of events of type $e \in\{R, M, 0\}$ in species $\mu$ (representing replacements, modifications, and no genetic changes, respectively), and

$$
p_{\mu, e}=\frac{n_{\mu, e}}{\sum_{e^{\prime}} n_{\mu, e^{\prime}}}, \quad \bar{p}_{e}=\frac{\sum_{\mu} n_{\mu, e}}{\sum_{\mu, e^{\prime}} n_{\mu, e^{\prime}}} .
$$

Equation (2a) quantifies the degree to which the observed replacement and modification rates deviate from a null model in which these events occur at a uniform rate across species. We assessed the significance of this deviation by comparing the observed value of $\Lambda$ to a null model in which the replacement and modification events were randomly permuted across all quasi-phaseable samples.

This defines a corresponding $P$-value,

$$
P=\operatorname{Pr}\left[\Lambda \geq \Lambda^{o b s}\right],
$$

which we estimated numerically from $n=10^{4}$ bootstrapped samples. We performed this test for the observed species counts $n_{\mu, e}$, as well as coarse-grained versions that merged the observed counts at the genus, family, or phylum levels (Fig. 1A,B).

The confidence intervals in Fig. 1 were obtained from the $\alpha / 2$ and $(1-\alpha) / 2$ percentiles of the posterior distribution of the underlying Poisson process,

$$
p\left(p_{\mu, e} \mid \vec{n}\right) \propto p_{\mu, e}^{n_{\mu, e}-1} e^{-p_{\mu, e} \sum_{e} n_{\mu, e}},
$$

as described in Ref. (4), with $\alpha=0.05$.

\section{Quantifying the relationship between community diversity and the rates of evolutionary modifi- cation}

We quantified the residual effects of community diversity on the rates of evolutionary modification using a logistic regression model, in which the probability of an evolutionary modification in species $\mu$ in community $c$ is given by

$$
\log \left(\frac{p_{\mu, c}}{1-p_{\mu, c}}\right)=\beta_{0}+\vec{\beta}_{P} \cdot \vec{P}_{\mu}+\beta_{H} H_{c}^{0}
$$

where $\vec{P}_{\mu}$ is an indicator variable giving the phylum of species $\mu$, and $H_{c}^{0}$ is the Shannon entropy of the surrounding community,

$$
H_{c}^{0}=-\sum_{\nu} f_{\nu, 0} \log _{2} f_{\nu, 0}
$$

which was estimated from the relative abundances of the species at the initial timepoint. For simplicity, we only examined species in the Bacteroidetes and Firmicutes phyla, which constitute the vast majority of our data (Fig. 1B). Regression coefficients and $P$-values were estimated using the logistic regression routines in the statsmodels library (44) using default parameter values. Similar results were obtained when $H_{c}^{0}$ was replaced with a measure of species richness (Fig. S2). 


\section{Quantifying the overdispersion of genetic changes within hosts}

We quantified the residual effects of more general community properties on replacement and modification rates by examining the overdispersion of genetic changes within a given host community. For each pair of timepoints, we calculated the total number of quasi-phaseable populations that experienced a replacement or modification event, and examined the distribution of these counts across our dataset (Fig. 1D). We compared this distribution to a null model in which the replacement and modification events were randomly permuted across quasi-phaseable populations of the same species. This permutation scheme eliminates any correlations between the genetic changes in different resident populations, but preserves the heterogeneity in the number and types of quasi-phaseable populations in different hosts, and the total number of replacement and modification events in different species.

\section{Quantifying shifts in ecological structure over time}

We quantified the changes in ecological structure between a pair of timepoints using the Jensen-Shannon divergence,

$$
\begin{aligned}
J S D & =\sum_{\mu}\left[f_{\mu, 0} \log _{2}\left(\frac{2 f_{\mu, 0}}{f_{\mu, 0}+f_{\mu, 1}}\right)+f_{\mu, 1} \log _{2}\left(\frac{2 f_{\mu, 1}}{f_{\mu, 0}+f_{\mu, 1}}\right)\right], \\
& =\sum_{\mu} \bar{f}_{\mu}\left[\left(\frac{2}{1+r_{\mu}}\right) \log \left(\frac{2}{1+r_{\mu}}\right)+\left(\frac{2}{1+r_{\mu}^{-1}}\right) \log \left(\frac{2}{1+r_{\mu}^{-1}}\right)\right],
\end{aligned}
$$

where $f_{\mu, 0}$ and $f_{\mu, 1}$ are the relative abundances of species $\mu$ at the initial and final timepoints, $\bar{f}_{\mu}=\left(f_{\mu, 0}+f_{\mu, 1}\right) / 2$ is the average relative abundance, and $r_{\mu}=f_{\mu, 1} / f_{\mu, 0}$ is the fold change between the two time points. The Jensen-Shannon distance is (Fig. 2D) is defined as the square root of the Jensen-Shannon divergence $(d=\sqrt{J S D})$.

We quantified the differences between the distributions of Jensen-Shannon distances in Fig. 2D using a one-sided Kolmogorov-Smirnov (KS) test,

$$
D_{e}=\max _{d}\left\{\hat{S}_{e}(d)-\hat{S}_{0}(d)\right\}
$$

where $\hat{S}_{e}(d)$ is the fraction of communities of type $e \in\{R, M, 0\}$ with Jensen-Shannon distance larger than $d$. Larger values of $D_{e}$ indicate an enrichment of larger Jensen-Shannon distances, relative to communities with no genetic changes. We assessed the significance of these differences by comparing the observed $D_{e}$ values to a null model similar to Fig. 1D, in which the replacement and modification events were randomly permuted across quasi-phaseable populations of the same species. As above, this permutation scheme preserves the heterogeneous opportunities for replacement and modification events in different hosts, the observed distribution of Jensen-Shannon distances, and any correlations between the two. This defines a corresponding $P$-value,

$$
P=\operatorname{Pr}\left[D_{e} \geq D_{e}^{o b s}\right],
$$

which we estimated numerically from $n=10^{4}$ bootstrapped samples. For completeness, we used a similar approach to compare the overall change in Shannon diversity between the two timepoints (Fig. S5). We observed a much weaker correlation with the number of replacement or modification events, suggesting that the signals in Fig. 2 cannot be explained by diversity fluctuations alone.

In addition to these entropy-based metrics, we also quantified shifts in community structure by examining extinction events among highly abundant species (Fig. 2G). In this calculation, a species 
was counted as going extinct if it transitioned from an initial relative abundance $>1 \%$ to a final relative abundance $<0.01 \%$. We compared the distributions of extinction events in Fig. $2 \mathrm{G}$ using a similar KS test as Fig. 2D, with the number of extinction events in a given community (Fig. S6) replacing the Jensen-Shannon distance in Eq. (7); we assessed the significance of these differences using the same permutation-based null model as above. We also compared these observations to the corresponding number of "invasion" events, defined as the time-reversed version of an extinction event above (Fig. S6). We quantified the asymmetry between extinctions and invasions by comparing them to a null model in which we randomly flipped the direction of time in each sample.

We used a similar approach to compare the distributions of focal species fold changes Fig. 3B, with the magnitude of the log fold change, $\left|\log \left(f_{\mu, 1} / f_{\mu, 0}\right)\right|$, replacing the Jensen-Shannon distance in Eq. (7). We quantified the excess of positive over negative changes (Fig. 3A) using a one-sided sign test. Similar results were obtained by comparing the fraction of positive changes in species with modifications vs no genetic changes using the permutation-based null model above $(P \approx 0.02)$.

\section{Decomposing the Jensen-Shannon divergence}

The linear sum in Eq. (6b) provides a natural way to decompose the Jensen-Shannon divergence into contributions from different species. For example, the fraction of Jensen-Shannon divergence "explained" by a subset of species $\mu \in \mathcal{S}$ can be defined as

$$
p(\mathcal{S})=\frac{\sum_{\mu \in \mathcal{S}} \bar{f}_{\mu}\left[\left(\frac{2}{1+r_{\mu}}\right) \log \left(\frac{2}{1+r_{\mu}}\right)+\left(\frac{2}{1+r_{\mu}^{-1}}\right) \log \left(\frac{2}{1+r_{\mu}^{-1}}\right)\right]}{J S D} .
$$

Figure 2E shows the percent JSD explained by the subset of strains with a minimum fold change $r$, by defining

$$
\mathcal{S}(r)=\left\{\mu:\left|\log r_{\mu}\right| \geq|\log r|\right\} .
$$

Similarly, Fig. 3 shows the percent JSD explained by a single focal species (Fig. 3C), the entire set of focal species (Fig. 3E), or all species in the same family as one of the focal species (Fig. 3F).

In the latter two cases, we compared these distributions to a null model in which the same number of replacement or modification events were redrawn from the same subset of hosts, by weighting each resident population by the empirical rate of replacement or modification events in that species across our entire dataset (Fig. 3E,F). We assessed the significance of these differences using the mean of this distribution as a test statistic. 

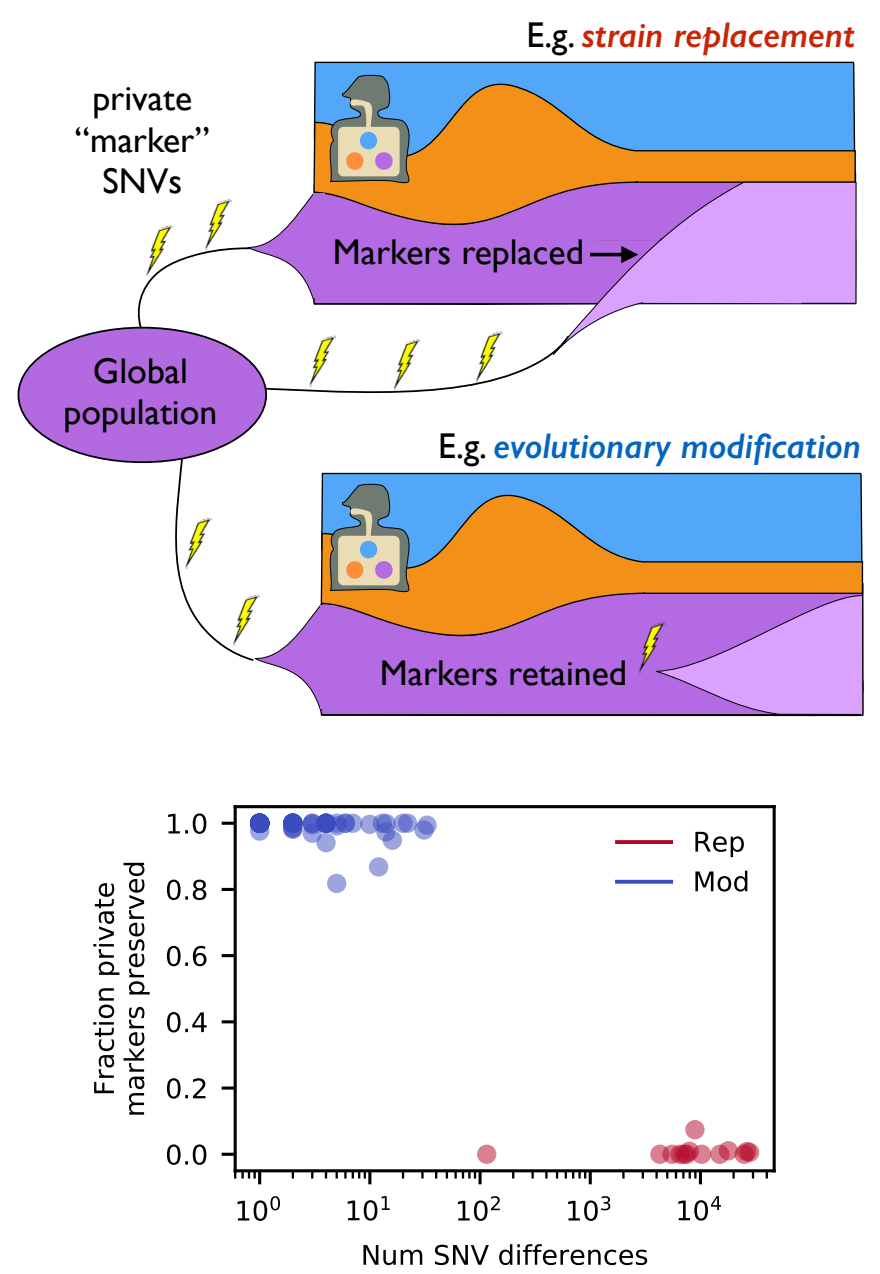

Figure S1: Schematic illustration of strain replacement and evolutionary modification events within a host. In the former case, private marker SNVs that are fixed in the resident population at the initial timepoint are replaced when an external strain sweeps through the population $(4,9)$. In contrast, private marker SNVs are preserved in an evolutionary modification event, when mutations accumulate on the background of the resident strain. Bottom panel shows the fraction of private marker SNVs preserved as a function of the total number of SNV differences for all of the populations in our study with at least 10 private marker SNVs. The sharp transition at $\approx 100$ SNVs motivates our operational definition of a strain replacement event as one with $>100$ SNV differences between timepoints (Methods). 


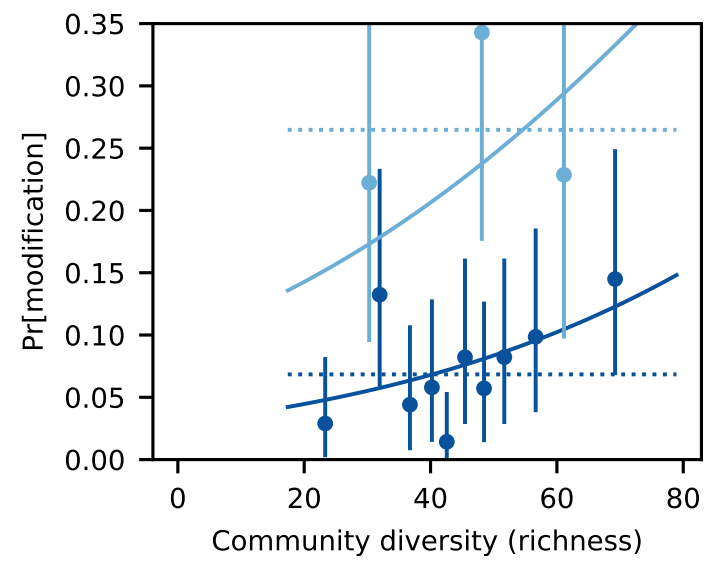

Figure S2: An analogous version of Fig. 1C using species richness as a measure of community diversity. Richness was calculated as $\mathcal{S}=\sum_{\mu}\left[1-\exp \left(-f_{\mu} / f_{0}\right)\right]$ with $f_{0}=10^{-3}$, which approximates the number of species with relative abundance $\gtrsim 10^{-3}$.
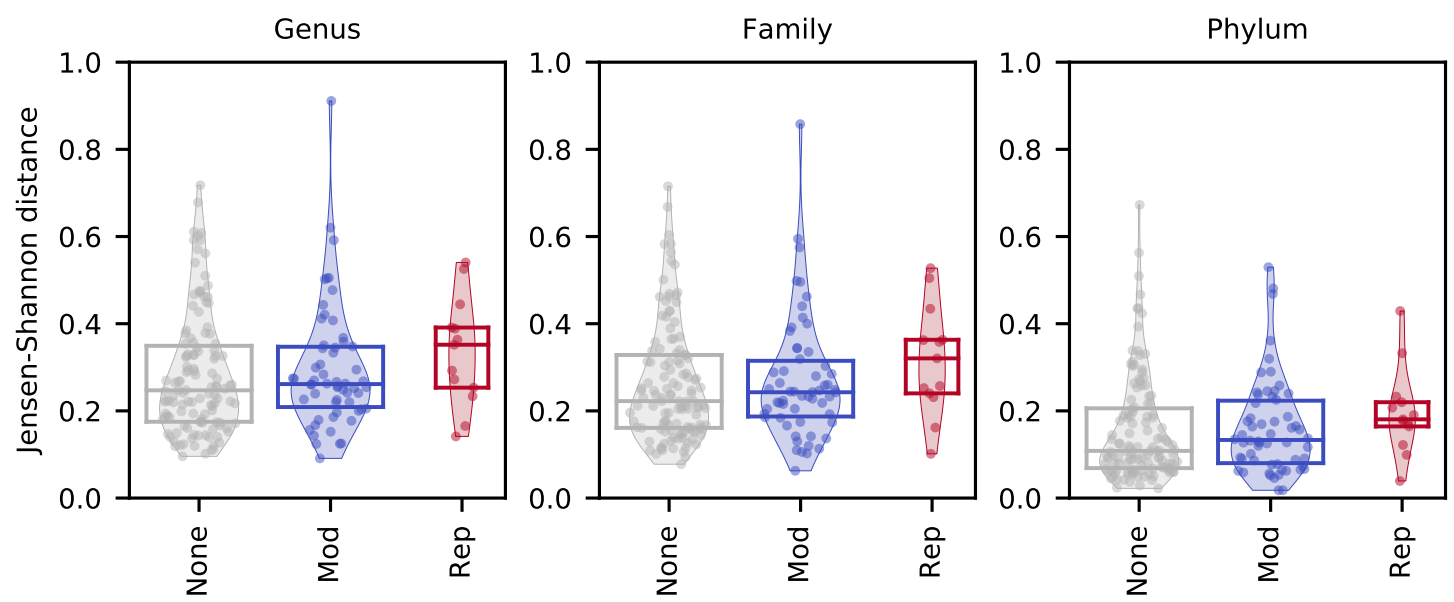

Figure S3: Analogous versions of Fig. 2D using Jensen-Shannon distances computed from species abundances coarse-grained at the genus, family, and phylum levels. The corresponding $P$-values of the one-sided KS tests (Methods) are $P_{\text {mod }} \approx 0.01, P_{\text {rep }} \approx 0.01$ (genus-level), $P_{\text {mod }} \approx 0.02, P_{\text {rep }} \approx 0.001$ (family-level), $P_{\text {mod }} \approx 0.01, P_{\text {rep }} \approx 0.001$ (phylum-level). 


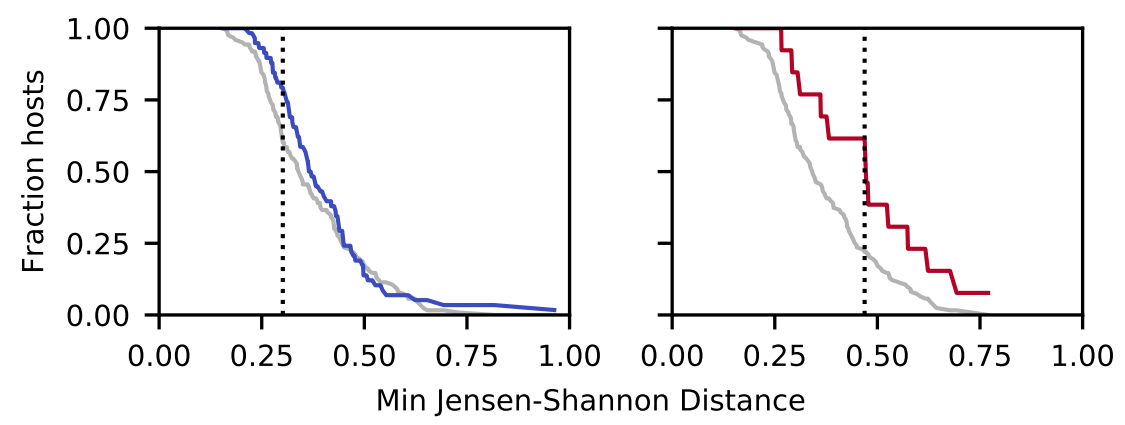

Figure S4: Survival distributions for the Jensen-Shannon distances in Fig. 2D, illustrating the onesided Kolmogorov-Smirnov (KS) test (Methods). The dashed line shows the location of the maximum deviation between the two deviations, which defines the KS statistic $D$ in Eq. (7).
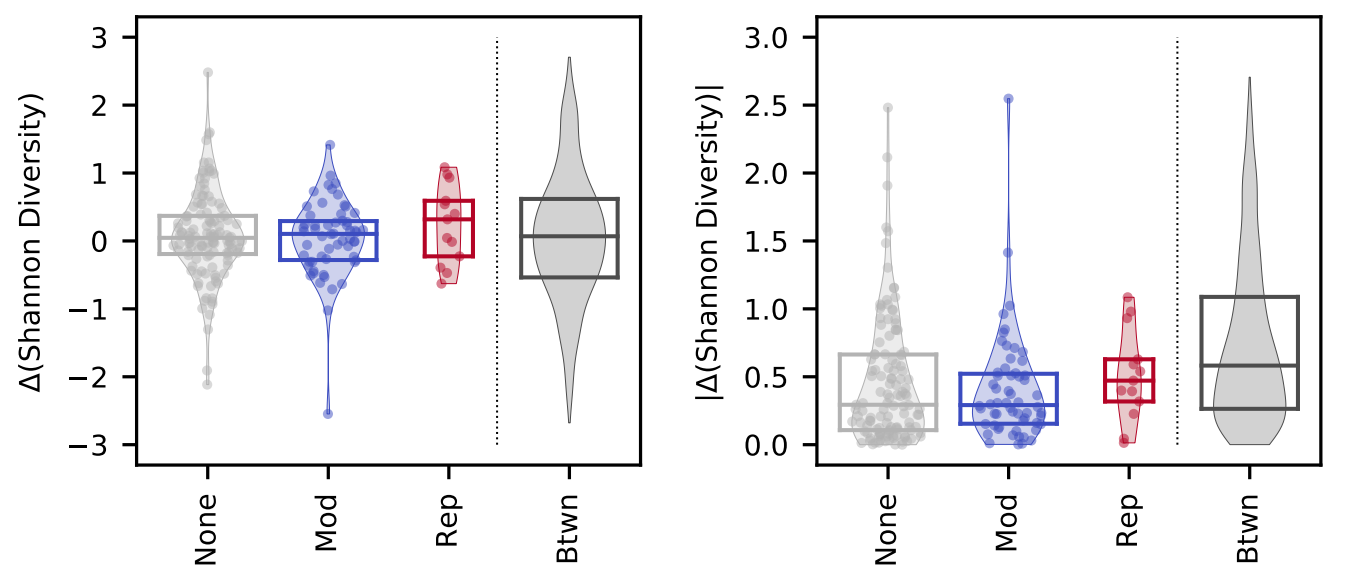

Figure S5: An analogous version of Fig. 2D using the change in Shannon diversity (left) or its absolute value (right) as an ecological distance metric. The only (marginally) significant difference occurs for the absolute value of the replacement events $(P \approx 0.05$, Methods); all other comparisons have $P>0.1$. 

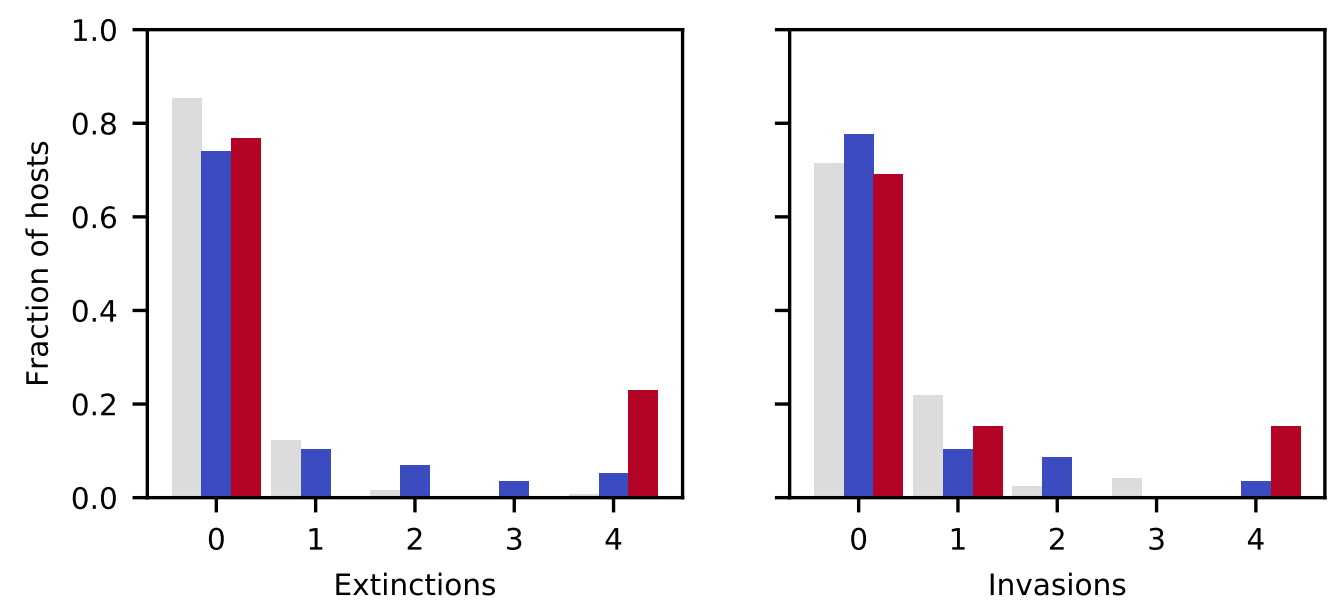

Figure S6: An analogous version of Fig. 2G showing "invasion" as well as "extinction" events of abundant species (Methods). Invasions are defined as the time-reversed version of an extinction event, so that the relative numbers of extinction and invasion events can be compared. In contrast to extinction events, replacement and modifications do not show a significantly increased number of invasions relative to hosts with no genetic changes $\left(P_{\text {rep }} \approx 0.08\right.$ and $P_{\text {mod }} \approx 0.2$, Methods). Interestingly, however, hosts with no genetic changes have a significantly increased fraction of invasions vs extinctions $(P \approx 0.01$, Methods), indicating a violation of time-reversal symmetry. 


\section{Text S1: Eco-evolutionary feedbacks in saturated resource competition models}

To develop intuition for the potential mechanisms that could produce the data in Figs. 1-3, we studied the eco-evolutionary correlations that arise in a simple mathematical model of an evolving microbial community. We focused on a simple class of resource competition models (32) of the form studied in Ref. (15) and other works $(33,45,46)$. We assume that microbial cells compete within a well-mixed, chemostat-like environment, in which $\mathcal{R}$ substitutable resources are supplied at constant flux, with $\beta_{i}$ denoting the fraction of biomass supplied in the form of resource $i$. We assume that species take up these resources at different genetically encoded rates, $r_{\mu, i}$. We previously showed (15) that it can be useful to decompose these uptake rates into a normalized resource strategy,

$$
\alpha_{\mu, i}=\frac{r_{\mu, i}}{\sum_{j} r_{\mu, j}},
$$

which represents the fraction of effort devoted to acquiring resources of type $i$, as well as a total uptake rate,

$$
X_{\mu}=\log \left(\sum_{j} \frac{r_{\mu, i}}{r_{0}}\right),
$$

which we will call the general fitness component. In this notation, the resulting ecological dynamics can then be written in a compact form,

$$
\frac{\partial f_{\mu}}{\partial t}=\left[-1+\sum_{i=1}^{\mathcal{R}} \frac{\beta_{i} \alpha_{\mu, i} e^{X_{\mu}}}{\sum_{\nu} \alpha_{\nu, i} e^{X_{\nu}} f_{\nu}}\right] f_{\mu}+\text { noise, }
$$

where $f_{\mu}$ denotes the relative abundance of species $\mu$ (15). At long times, these dynamics approach a unique stable fixed point (ecological equilibrium) with at most $\mathcal{R}$ coexisting species.

Here we focus on a scenario in which the community is already at its ecological equilibrium, and a new mutation occurs in one of the $\mathcal{S}$ resident species. In principle, this initial collection of species could be produced by a complex assembly process, involving a mixture of habitat filtering, host selection, and/or previous local evolution. Here, we will focus on the special case of a saturated community $(\mathcal{S}=\mathcal{R})$, where the ecological equilibria are particularly well characterized. We previously showed that the relative abundances in such an ecosystem are given by

$$
f_{\mu}=\sum_{i=1}^{\mathcal{R}} \frac{\beta_{i} \alpha_{i, \mu}^{-1}}{1+\sum_{\nu} \alpha_{i, \nu}^{-1}\left(e^{X_{\mu}-X_{\nu}}-1\right)},
$$

where $\alpha_{i, \mu}^{-1}$ is the inverse of $\alpha_{\mu, i}$. The normalization of the resource strategies $\left(\sum_{i} \alpha_{\mu, i}=1\right)$ implies that $\sum_{\mu} \alpha_{i, \mu}^{-1}=1$. Much of the relevant behavior can be observed for a special class of resource strategies,

$$
\alpha_{\mu, i}=\beta_{i}(1-\epsilon)+\epsilon \cdot \tilde{\alpha}_{\mu, i},
$$

where $\tilde{\alpha}_{\mu, i}$ is another normalized resource strategy and $\epsilon$ is a small parameter. In this case, the inverse of $\alpha_{\mu, i}$ is asymptotically given by

$$
\alpha_{i, \mu}^{-1}=\frac{\tilde{\alpha}_{i, \mu}^{-1}-\sum_{i} \beta_{i} \tilde{\alpha}_{i, \mu}^{-1}}{\epsilon}+\sum_{i} \beta_{i} \tilde{\alpha}_{i, \mu}^{-1}+\mathcal{O}(\epsilon) .
$$


If we define a corresponding rescaling of the overall fitness, $X_{\mu}=\epsilon^{2} \tilde{X}_{\mu}$, then we can expand Eq. (S1.4) in the limit of small $\epsilon$ to obtain

$$
f_{\mu}=\sum_{i} \beta_{i} \tilde{\alpha}_{i, \mu}^{-1}+\sum_{i, j, k, \nu} \beta_{i} \beta_{j} \beta_{k}\left(\tilde{\alpha}_{i, \mu}^{-1} \tilde{\alpha}_{i, \nu}^{-1}-\tilde{\alpha}_{j, \mu}^{-1} \tilde{\alpha}_{k, \nu}^{-1}\right) \tilde{X}_{\nu}+o(\epsilon)
$$

This expression can also be written in the compact form,

$$
f_{\mu}=\mathbb{E}_{\beta}\left[\alpha_{\mu}^{-1}\right]+\sum_{\nu} \operatorname{Cov}_{\beta}\left(\alpha_{\mu}^{-1}, \alpha_{\nu}^{-1}\right) X_{\mu}+o(\epsilon),
$$

where $\mathbb{E}_{\beta}\left[\alpha_{\mu}^{-1}\right]$ and $\operatorname{Cov}_{\beta}\left(\alpha_{\mu}^{-1}, \alpha_{\nu}^{-1}\right)$ denote particular moments of the inverse matrix:

$$
\begin{aligned}
\mathbb{E}_{\beta}\left[\alpha_{\mu}^{-1}\right] & \equiv \sum_{i} \beta_{i} \alpha_{i, \mu}^{-1}, \\
\operatorname{Cov}_{\beta}\left(\alpha_{\mu}^{-1}, \alpha_{\nu}^{-1}\right) & \equiv \sum_{i} \beta_{i} \alpha_{i, \mu}^{-1} \alpha_{i, \nu}^{-1}-\mathbb{E}_{\beta}\left[\alpha_{\mu}^{-1}\right] \cdot \mathbb{E}_{\beta}\left[\alpha_{\nu}^{-1}\right],
\end{aligned}
$$

which are weighted by the external resource supply rates $\beta_{i}$.

Within such a community, we wish to examine the invasion fitness and ecological impact of a mutation that changes the phenotype of a focal species $\left(\mu^{*}\right)$. These mutations can change the resource strategy $\alpha_{\mu, i}$, the overall fitness component $X_{\mu}$, or a combination of the two. We can write this as:

$$
X_{\mu^{*}} \rightarrow X_{\mu^{*}}+\Delta X, \quad \alpha_{\mu^{*}, i} \rightarrow \alpha_{\mu^{*}, i}+\gamma_{i},
$$

where $\gamma_{i}$ is normalized so that $\sum_{i} \gamma_{i}=0$. We previously showed (15) that the invasion fitness of such a mutation is given by

$$
S_{\mathrm{inv}}=\Delta X-\sum_{i=1}^{\mathcal{R}} \gamma_{i} \bar{X}_{i}
$$

where $\bar{X}_{i}=\sum_{\mu} \alpha_{i, \mu}^{-1} X_{\mu}$. By defining scaled versions of these quantities $\left(S_{\mathrm{inv}}=\epsilon^{2} \tilde{S}_{\mathrm{inv}}\right.$, $\Delta X=\epsilon^{2} \Delta \tilde{X}$, and $\gamma_{i}=\epsilon \tilde{\gamma}_{i}$ ), this reduces to

$$
\tilde{S}_{\mathrm{inv}} \approx \Delta \tilde{X}-\sum_{i, \mu} \tilde{\gamma}_{i} \tilde{\alpha}_{i, \mu}^{-1} \tilde{X}_{\mu}+o(\epsilon)
$$

in the limit that $\epsilon \ll 1$ (15). Note that this expression is independent of the external resource supply rates $\beta_{i}$. This shows that, in saturated communities at ecological equilibrium, the selection pressures on new mutations are independent of the external environment. This occurs because saturated communities can dynamically readjust their species composition in Eq. (S1.4) in such a way that the internal resource concentrations remain constant $(15,45)$. In this way, the collective behavior of the community will tend to shield it from any ecology-driven feedbacks, provided that the timescales of the external fluctuations are long compared to the internal equilibration time. This provides a proof-of-principle example of a large community in which only evolutionary feedbacks are possible. We will consider these feedbacks for different classes of mutations below.

Pure fitness mutations. We first consider the case where the mutation increases the overall fitness of the strain $(\Delta X>0)$ but leaves its resource strategy intact $\left(\gamma_{i}=0\right)$. The invasion fitness of such a mutation is simply given by

$$
\tilde{S}_{\mathrm{inv}}=\Delta \tilde{X} \quad \Longrightarrow \quad S_{\mathrm{inv}}=\Delta X
$$


If this mutation establishes, it will outcompete its ancestor and alter the ecological equilibrium between species. For sufficiently small $\Delta \tilde{X}$, none of the other species will go extinct, and the new equilibrium can be calculated from Eq. (S1.4) by perturbing $\tilde{X}_{\mu} \rightarrow \tilde{X}_{\mu}+\Delta \tilde{X} \delta_{\mu, \mu^{*}}$. The corresponding relative abundance changes are given by

$$
\Delta f_{\mu}=\Delta \tilde{X} \times \sum_{i, j, k} \beta_{i} \beta_{j} \beta_{k}\left(\tilde{\alpha}_{i, \mu}^{-1} \tilde{\alpha}_{i, \mu^{*}}^{-1}-\tilde{\alpha}_{j, \mu}^{-1} \tilde{\alpha}_{k, \mu^{*}}^{-1}\right)
$$

which can also be written as

$$
\Delta f_{\mu}=S_{\mathrm{inv}}\left(\frac{\operatorname{Cov}_{\beta}\left(\tilde{\alpha}_{\mu}^{-1}, \tilde{\alpha}_{\mu^{*}}^{-1}\right)}{\epsilon^{2}}\right),
$$

where $\operatorname{Cov}_{\beta}\left(\tilde{\alpha}_{\mu}^{-1}, \tilde{\alpha}_{\mu^{*}}^{-1}\right)$ is defined as in Eq. (S1.10) above. This shows that for a given invasion fitness $S_{\text {inv }}$ (e.g. as measured in metagenomic sequencing), larger shifts in frequency occur in communities with a larger degree of metabolic overlap (smaller $\epsilon$ ). Conversely, for a fixed set of resource strategies, larger invasion fitnesses result in larger ecological perturbations. Furthermore, since

$$
\operatorname{Cov}_{\beta}\left(\tilde{\alpha}_{\mu^{*}}^{-1}, \tilde{\alpha}_{\mu^{*}}^{-1}\right)=\operatorname{Var}_{\beta}\left(\tilde{\alpha}_{\mu^{*}}^{-1}\right) \geq 0
$$

Eq. (S1.16) shows that the shift in the focal strain is always positive for a pure fitness mutation. The ratio of the shifts in frequency can be written as

$$
\frac{\Delta f_{\mu}}{\Delta f_{\mu^{*}}}=\frac{\operatorname{Cov}_{\beta}\left(\tilde{\alpha}_{\mu}^{-1}, \tilde{\alpha}_{\mu^{*}}^{-1}\right)}{\operatorname{Var}_{\beta}\left(\tilde{\alpha}_{\mu^{*}}^{-1}\right)},
$$

which is independent of $S_{\mathrm{inv}}, \epsilon$, and $X_{\mu}$, and depends only on the strategy covariance matrices defined above. The right hand side of Eq. (S1.18) is reminiscent of some classical measures of niche overlap computed from the correlations of resource utilization vectors (47). In this case, however, because the covariances depend on the inverse matrix $\left(\alpha_{i, \mu}^{-1}\right)$ rather than the resource strategies themselves, the abundance fluctuations will generally depend on the resource strategies of the entire community (as opposed to $\mu$ and $\mu^{*}$ alone).

Pure strategy mutations. We next consider the case where the mutation alters the resource strategy of the strain $\left(\gamma_{i} \neq 0\right)$ but leaves its overall fitness component intact $(\Delta X=0)$. Equation (S1.13) shows that the invasion fitness of such a mutation is given by

$$
\tilde{S}_{\mathrm{inv}} \approx-\sum_{i, \mu} \tilde{\gamma}_{i} \tilde{\alpha}_{i, \mu}^{-1} \tilde{X}_{\mu}
$$

This shows that only a subset of these mutations will be favored by selection $\left(S_{\mathrm{inv}}>0\right)$. As above, we wish to understand how a successful strategy mutation changes the ecological equilibrium at steady state. In the limit of small $\tilde{\gamma}_{i}$, the mutation will outcompete its parent strain and lead to small changes in the frequencies of the other strains. This results in a new set of resource strategy vectors,

$$
\tilde{\alpha}_{\mu, i}^{\prime}=\tilde{\alpha}_{\mu, i}+\gamma_{i} \delta_{\mu, \mu^{*}}
$$

We can calculate the new equilibrium frequencies from Eq. (S1.4) with the help of the identity,

$$
\left(\tilde{\alpha}_{i, \mu}^{\prime}\right)^{-1} \approx \tilde{\alpha}_{i, \mu}^{-1}-\sum_{j, \nu} \tilde{\alpha}_{i, \nu}^{-1} \tilde{\gamma}_{j} \delta_{\nu, \mu^{*}} \tilde{\alpha}_{j, \mu}^{-1} \approx \tilde{\alpha}_{i, \mu}^{-1}-\tilde{\alpha}_{i, \mu^{*}}^{-1} \sum_{j} \tilde{\gamma}_{j} \tilde{\alpha}_{j, \mu}^{-1}
$$


Substituting into Eq. (S1.4), we obtain

$$
\begin{aligned}
\Delta f_{\mu}=- & f_{\mu^{*}}^{0} \sum_{j} \tilde{\gamma}_{j} \tilde{\alpha}_{j, \mu}^{-1}-\sum_{i, j, \nu} \beta_{i} \tilde{\alpha}_{i, \mu}^{-1} \tilde{\alpha}_{i, \mu^{*}}^{-1} \cdot \tilde{\gamma}_{j} \tilde{\alpha}_{j, \nu}^{-1} \tilde{X}_{\nu}-\sum_{i, j, \nu} \beta_{i} \tilde{\alpha}_{i, \nu}^{-1} \tilde{\alpha}_{i, \mu^{*}}^{-1} \cdot \tilde{\gamma}_{j} \tilde{\alpha}_{j, \mu}^{-1} \tilde{X}_{\nu} \\
& +\sum_{\nu, j} f_{\mu^{*}}^{0} f_{\nu}^{0} \tilde{\gamma}_{j} \tilde{\alpha}_{j, \mu}^{-1} \tilde{X}_{\nu}+\sum_{\nu, j} f_{\mu^{*}}^{0} f_{\mu}^{0} \tilde{\gamma}_{j} \tilde{\alpha}_{j, \nu}^{-1} \tilde{X}_{\nu}+\mathcal{O}\left(\tilde{\gamma}^{2}\right)
\end{aligned}
$$

where $f_{\mu}^{0}=\mathbb{E}_{\beta}\left[\alpha_{\mu}^{-1}\right]$ is the equilibrium frequency in the absence of any fitness differences $\left(X_{\mu}=0\right)$. Using the definition of the invasion fitness in Eq. (S1.19), we can write expression in the compact form,

$$
\Delta f_{\mu}=-f_{\mu^{*}} \sum_{j} \tilde{\gamma}_{j} \tilde{\alpha}_{j, \mu}^{-1}+\tilde{S}_{\text {inv }}\left[\sum_{i, j} \beta_{i} \beta_{j}\left(\tilde{\alpha}_{i, \mu}^{-1} \tilde{\alpha}_{i, \mu^{*}}^{-1}-\tilde{\alpha}_{i, \mu}^{-1} \tilde{\alpha}_{j, \mu^{*}}^{-1}\right)\right] .
$$

We can then turn this expression around to solve for $\tilde{\gamma}_{i}$ as a function of $\Delta f_{\mu}$ :

$$
\tilde{\gamma}_{i}=-\frac{1}{f_{\mu^{*}}} \sum_{\mu} \Delta f_{\mu} \tilde{\alpha}_{\mu, i}+\frac{\tilde{S}_{\mathrm{inv}}}{f_{\mu^{*}}} \sum_{\mu} \tilde{\alpha}_{\mu, i}\left[\sum_{i, j} \beta_{i} \beta_{j}\left(\tilde{\alpha}_{i, \mu}^{-1} \tilde{\alpha}_{i, \mu^{*}}^{-1}-\tilde{\alpha}_{i, \mu}^{-1} \tilde{\alpha}_{j, \mu^{*}}^{-1}\right)\right] .
$$

Plugging back in to our expression for $\tilde{S}_{\text {inv }}$ in Eq. (S1.19), we have a self-consistent solution for $\tilde{S}_{\text {inv }}$ :

$$
\begin{aligned}
\tilde{S}_{\mathrm{inv}} & =\frac{1}{f_{\mu^{*}}} \sum_{i, \nu, \mu} \tilde{\alpha}_{i, \nu}^{-1} X_{\nu} \Delta f_{\mu} \tilde{\alpha}_{\mu, i}-\frac{\tilde{S}_{\mathrm{inv}}}{f_{\mu^{*}}} \sum_{i, \nu, \mu} \tilde{\alpha}_{i, \nu}^{-1} X_{\nu} \tilde{\alpha}_{\mu, i}\left[\sum_{i, j} \beta_{i} \beta_{j}\left(\tilde{\alpha}_{i, \mu}^{-1} \tilde{\alpha}_{i, \mu^{*}}^{-1}-\tilde{\alpha}_{i, \mu}^{-1} \tilde{\alpha}_{j, \mu^{*}}^{-1}\right)\right] \\
& =\frac{1}{f_{\mu^{*}}} \sum_{\mu} \Delta f_{\mu} \tilde{X}_{\mu}-\frac{\tilde{S}_{\mathrm{inv}}}{f_{\mu^{*}}} \sum_{\mu} X_{\mu}\left[\sum_{i, j} \beta_{i} \beta_{j}\left(\tilde{\alpha}_{i, \mu}^{-1} \tilde{\alpha}_{i, \mu^{*}}^{-1}-\tilde{\alpha}_{i, \mu}^{-1} \tilde{\alpha}_{j, \mu^{*}}^{-1}\right)\right]
\end{aligned}
$$

Solving for $\tilde{S}_{\mathrm{inv}}$ and converting back to unscaled fitnesses, we find that

$$
S_{\mathrm{inv}}=\frac{\sum_{\mu} \Delta f_{\mu} \cdot X_{\mu}}{2 f_{\mu^{*}}-f_{\mu^{*}}^{0}}
$$

Note that the sign of this expression depends on the focal species only through the factor $2 f_{\mu^{*}}-f_{\mu^{*}}^{0}$, which is independent of the new mutation. When $2 f_{\mu^{*}}-f_{\mu^{*}}^{0}>0$, natural selection will favor mutations that increase the community-level quantity $\sum_{\mu} f_{\mu} \cdot X_{\mu}$, even if the focal species itself declines in abundance. Conversely, if $2 f_{\mu^{*}}-f_{\mu^{*}}^{0}<0$, natural selection will favor mutations that lead to lower values of $\sum f_{\mu} \cdot X_{\mu}$, independent of the focal species change.

General mutations. We can combine the results above to understand what happens for a general mutation. At linear order, we still obtain

$$
\Delta f_{\mu}=-f_{\mu^{*}} \sum_{j} \tilde{\gamma}_{j} \tilde{\alpha}_{j, \mu}^{-1}+\tilde{S}_{\mathrm{inv}}\left[\sum_{i, j} \beta_{i} \beta_{j}\left(\tilde{\alpha}_{i, \mu}^{-1} \tilde{\alpha}_{i, \mu^{*}}^{-1}-\tilde{\alpha}_{i, \mu}^{-1} \tilde{\alpha}_{j, \mu^{*}}^{-1}\right)\right]
$$

where $\tilde{S}_{\text {inv }}$ now includes the contribution from $\Delta \tilde{X}$. Solving for $\tilde{\gamma}_{i}$ and plugging into Eq. (S1.19), we obtain

$$
\tilde{S}_{\mathrm{inv}}=\Delta \tilde{X}+\frac{1}{f_{\mu^{*}}} \sum_{\mu} \Delta f_{\mu} \tilde{X}_{\mu}-\frac{\tilde{S}_{\mathrm{inv}}}{f_{\mu^{*}}} \sum_{\mu} X_{\mu}\left[\sum_{i, j} \beta_{i} \beta_{j}\left(\tilde{\alpha}_{i, \mu}^{-1} \tilde{\alpha}_{i, \mu^{*}}^{-1}-\tilde{\alpha}_{i, \mu}^{-1} \tilde{\alpha}_{j, 1}^{-1}\right)\right]
$$


which yields the general expression,

$$
S_{\mathrm{inv}}=\frac{\Delta X f_{\mu^{*}}+\sum_{\mu} \Delta f_{\mu} X_{\mu}}{2 f_{\mu^{*}}-f_{\mu^{*}}^{0}},
$$

quoted in Eq. (1) in the main text.

Example for two species. We can gain some further intuition for this result by focusing on the case where $\mathcal{S}=\mathcal{R}=2$. Without loss of generality, we can set $\mu^{*}=1$. In this case, the inverse resource strategy matrix can be computed exactly:

$$
\alpha_{i, \mu}^{-1}=\frac{1}{\Delta \alpha}\left(\begin{array}{cc}
1-\alpha_{2} & -\left(1-\alpha_{1}\right) \\
-\alpha_{2} & \alpha_{1}
\end{array}\right)
$$

so that

$$
\begin{gathered}
S_{\mathrm{inv}}=s-\frac{\gamma \Delta X}{\Delta \alpha}, \\
f_{1}^{0}=\sum_{i} \beta_{i} \alpha_{i, 1}^{-1}=\frac{\beta-\alpha_{2}}{\Delta \alpha},
\end{gathered}
$$

and

$$
f_{1}=\frac{\beta-\alpha_{2}}{\Delta \alpha}+\frac{\beta(1-\beta) \Delta X}{\Delta \alpha^{2}}
$$

The change in frequency from an infinitesimal $\gamma$ is given by

$$
\Delta f \approx f_{1}^{0} \cdot \frac{-\gamma}{\Delta \alpha}+\frac{\beta(1-\beta) \Delta X}{\Delta \alpha^{2}} \frac{-2 \gamma}{\Delta \alpha}
$$

or

$$
\Delta f=-\left(2 f_{1}-f_{1}^{0}\right) \frac{\gamma}{\Delta \alpha}
$$

These results show that for a fixed $\gamma$, the fitness landscape is independent of the sign of $2 f_{1}-f_{1}^{0}$. If $\Delta X>0$, then there is always selection pressure for $\gamma>0$. In this case, $f_{1}>f_{1}^{0}$, so the corresponding change $\Delta f$ is always positive. Conversely, if $\Delta X<0$, then there is always selection pressure for $\gamma<0$. In this case, the sign of $\Delta f$ depends on $f_{1}$ vs $f_{1}^{0}$. If $f_{1}>f_{1}^{0} / 2$, then the mutation leads to $\Delta f<0$, and an increase in $\sum_{\mu} f_{\mu} X_{\mu}$. However, if $f_{1}<f_{1}^{0} / 2$, then the mutation leads to $\Delta f>0$, and a decrease in the community-wide $\sum_{\mu} f_{\mu} X_{\mu}$.

Limitations and future directions. While the above results provide a useful proof-of-principle demonstration for the evolution-driven feedbacks that can emerge in simple ecological models, they have several notable limitations. Our present analysis has only considered the simplest case of small-effect mutations in saturated communities. Further work is necessary to understand how these results extend to non-saturated communities (45), and to incorporate important factors like cross-feeding $(34,35)$ and spatial structure within the gut $(36)$. Perhaps more importantly, our current results only show that it is theoretically possible to have a beneficial mutation with a given set of $\Delta f_{\mu}$ values. Whether this happens in practice depends on whether the corresponding values of $\gamma_{i}$ can be produced by mutations in one of the resident strains. The answer to this question requires additional 
assumptions about the genetic architecture of the resource uptake phenotypes, which is poorly constrained by existing data. Our results suggest that it would be interesting to map out the accessible ecological perturbations in future experiments, e.g. by measuring the joint distribution of $S_{\text {inv }}$ and $\Delta f_{\mu}$ values by mutating or swapping strains in large synthetic communities (38). This would constitute a eco-evolutionary analogue of the distribution of fitness effects that plays a central role in evolutionary genetics (48). 